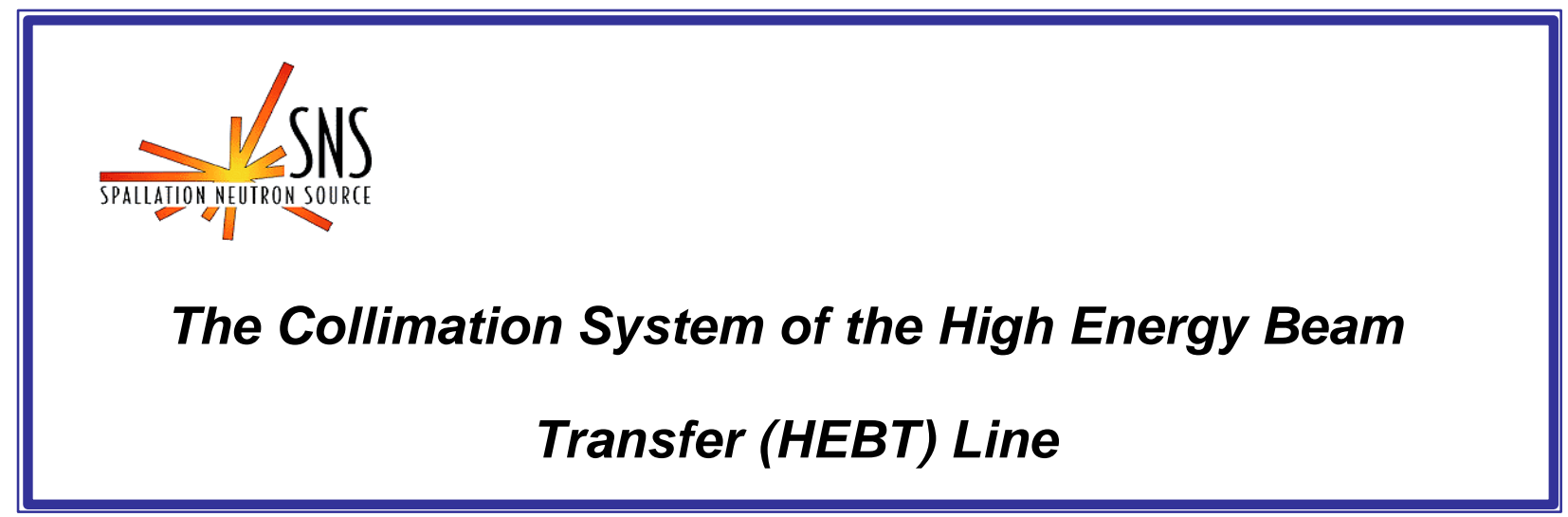

BNL/SNS TECHNICAL NOTE

NO. 097

N. Catalan-Lasheras, D. Raparia

July 27, 2001

COLLIDER-ACCELERATOR DEPARTMENT BROOKHAVEN NATIONAL LABORATORY

UPTON, NEW YORK 11973 


\title{
The Collimation System of the High Energy Beam Transfer (HEBT) Line.
}

\author{
N. Catalan-Lasheras and D. Raparia
}

July 27, 2001

\section{Introduction}

The High Energy Beam Transfer (HEBT) line of the SNS complex [1], matches the linac to the ring acceptance and assures the final quality of the beam. Besides diagnostics that characterize the beam, a collimation system is provided to protect the line and RF cavities and to avoid excessive transverse and longitudinal halo transported into the ring. The transverse system consists of two collimators $[2,3,4]$ of fixed aperture preceded by horizontal and vertical pair of striper foils working as primary collimators. A longitudinal collimation system is also provided in the achromat with a pair of facing striper foils followed by an absorber.

The last technical note on the HEBT line collimation system [5] described the modifications in the line optics and the reduction of the number collimators in the HEBT. Since then, the collimation system has been repositioned to increase the collimator aperture and to improve the absorption efficiency. New locations for the collimators and foils have been identified for optimum protection. The aperture of the foil of the longitudinal cleaning system has been revisited and the final efficiency estimated. In this note, we report the main reasons for this changes and compute the efficiency of the redesigned collimation systems.

\section{HEBT transverse collimation}

The rms emittance of the beam coming from the linac is expected to be small $\left(\epsilon_{r m s}=0.5 \pi \mathrm{mm} \cdot \mathrm{mrad}\right.$ normalised) but large tails in the distribution may contain a fraction of the beam larger than $10^{-4}$. Fig. 1 shows the beam fraction contained in the tails of a gaussian beam depending on the beam rms emittance. A factor of two in the beam emittance coming from the linac yields an increase of three orders of magnitude in the halo population. With a beam power of up to $2 \mathrm{MW}$ and beam losses spreading along few meters, the residual radiation does not satisfy the hands-on maintenance criteria. We need to provide a transverse collimation system upstream in the line to prevent losses on the achromat, cavities and injection in the ring. The minimum aperture in the HEBT line is given by the two RF cavities used for spreading the beam momentum and correcting the central energy [1]. The cavities acceptance is $A_{x}=26 \pi \mathrm{mm} \cdot \mathrm{mrad}$. With a small sets of collimators in each plane, the main function is to prevent the transport along the line of $H^{-}$ions with emittance larger than the line acceptance. Collimators provide little additional shaping capability.

We have at our disposal two sets of collimators in each plane. After the redesign of the optics in the line [5], the phase advance between collimators had been reduced to 45 degrees. To protect 


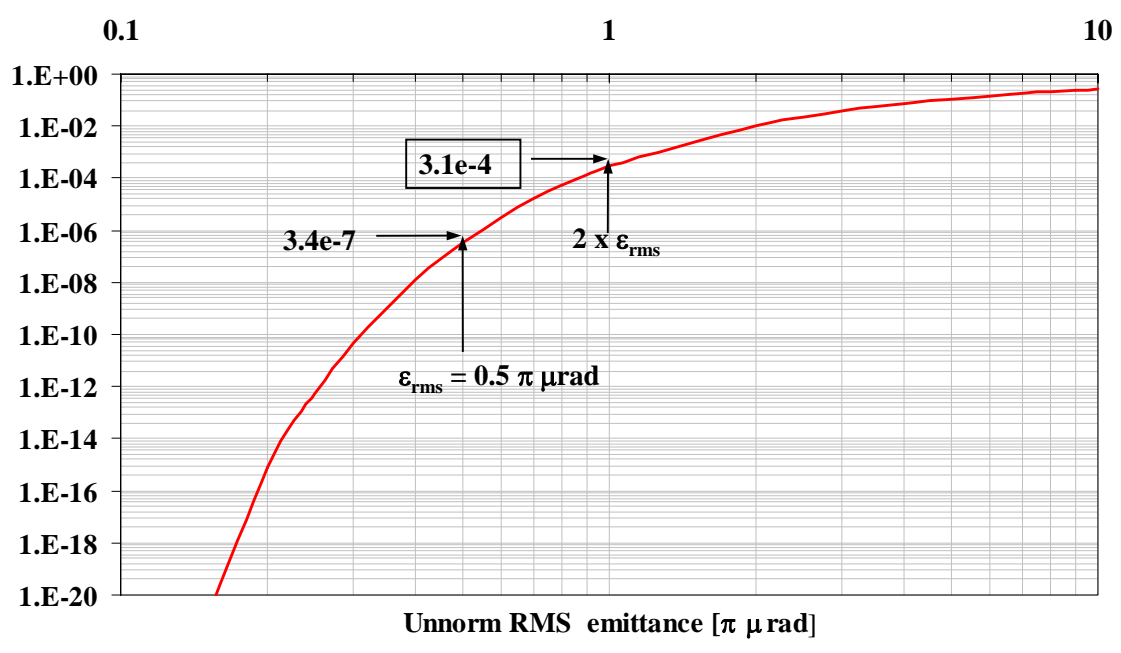

Figure 1: Fraction of the beam exceeding a fixed aperture in the HEBT line. The aperture is chosen to match $13 \pi \mathrm{mm} \cdot \mathrm{mrad}$, half of the HEBT acceptance and equal to the collimator acceptance.

the cavities, the final emittance of any escaping particle must be less than $A_{H E B T}=26 \pi \mathrm{mm} \cdot \mathrm{mrad}$. As it can be seen from Fig. 2 collimators located at 90 degrees of phase advance apart provide the most efficient cut of the phase space. With $\Delta \mu=90^{\circ}$ the aperture of the collimators can be set at $13 \pi \mathrm{mm} \cdot \mathrm{mrad}$. For a system with only $45^{\circ}$ of phase advance, the aperture has to be set at $3 \pi \mathrm{mm} \cdot \mathrm{mrad}$. This requirement, unnecessarily reduces the aperture of the line and increases the heat load of the collimators

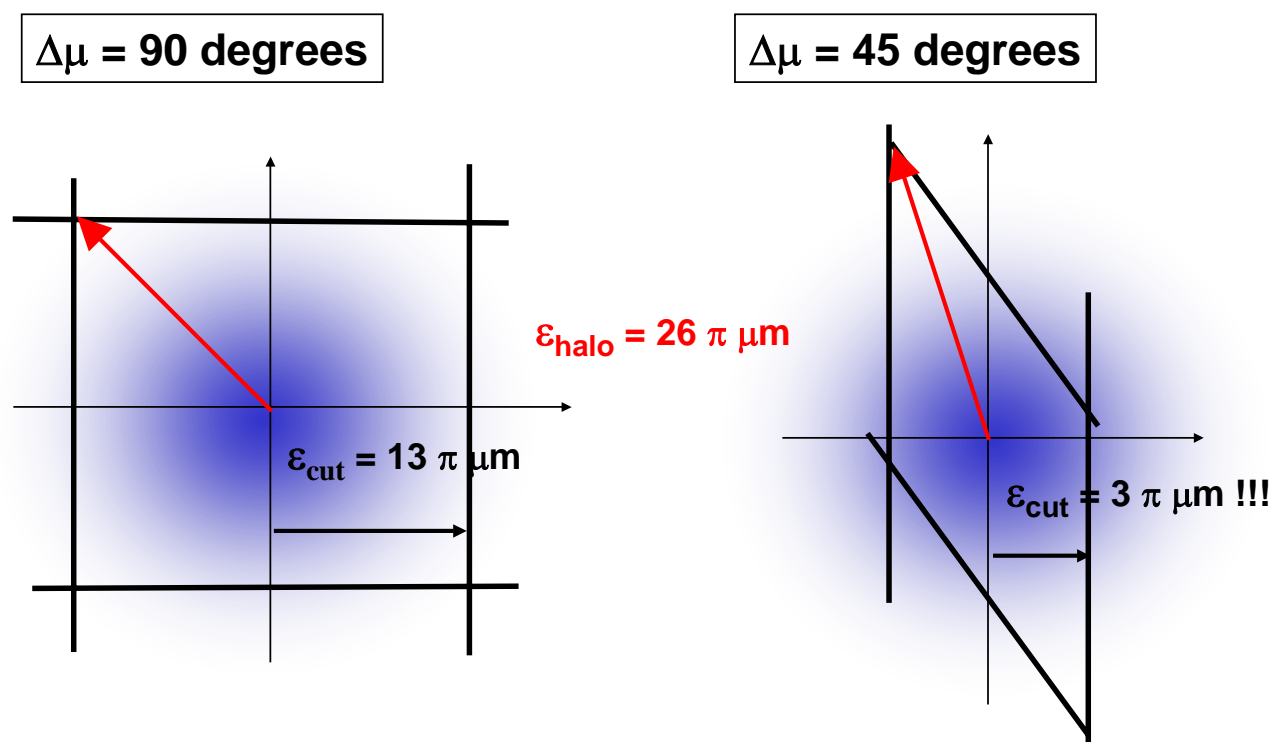

Figure 2: Cuts induced by the striper foils in normalized phase-space for two systems at 90 and 45 degrees of phase advance, respectively. The maximum extent of the escaping halo is required to be $26 \pi \mathrm{mm} \cdot \mathrm{mrad}$ in both cases. 


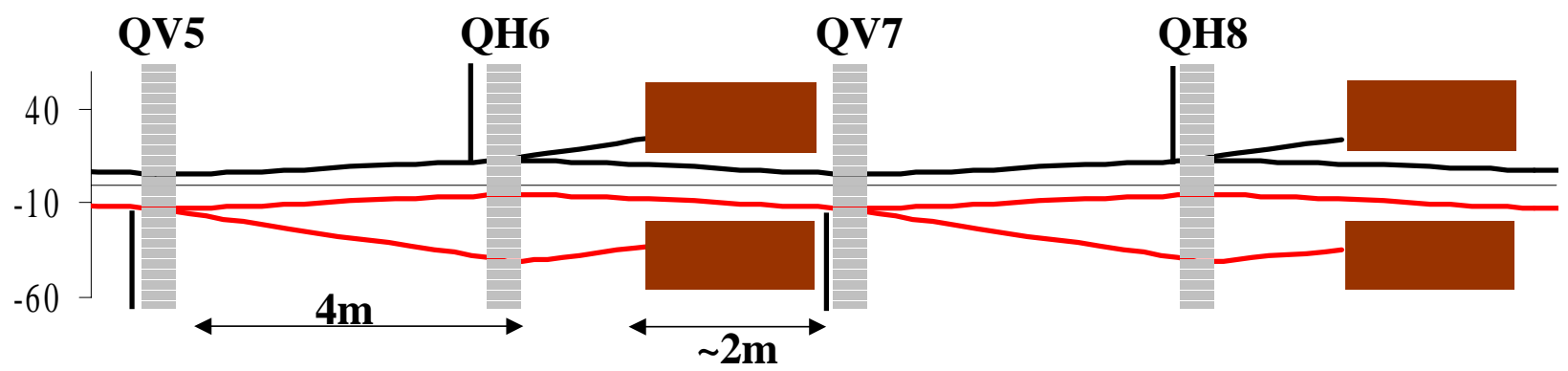

Figure 3: Layout of the HEBT collimation system and proton beam trajectories. Top half corresponds to the horizontal plane while the vertical is represented by the bottom half.

\subsection{System layout}

To remove the transverse halo from the beam, we introduce adjustable carbon foils in the path of the beam to intercept the tails. After the passage of the $H^{-}$ion through the foil, the two electrons are removed and the $H^{-}$focusing lattice deviates the proton tails into large massive absorbers [4].

The horizontal and vertical striping foils are located at the maximum of the beta function in each plane to enhance the resolution of the system. However, the actual position along the drift does not affect the final trajectory and the collimation efficiency. Once the position and acceptance of the foils is fixed, the aperture and position of the absorber is decided by the focusing lattice and the required impact parameter.

We transported a proton beam created at each foil with any emittance value in the vertical plane. Fig. 4 shows the projection in real space of the protons generated at both horizontal and vertical collimator at their passage through the quadrupoles and at the front face of the collimator. The minimal impact parameters on the absorbers of the proton beam produced in each of the stripers is shown in Tab. 1. The beam clears the quadrupoles aperture and hits the absorber with a large impact parameter. Large aperture quadrupoles are not necessary with this configuration. The location of the
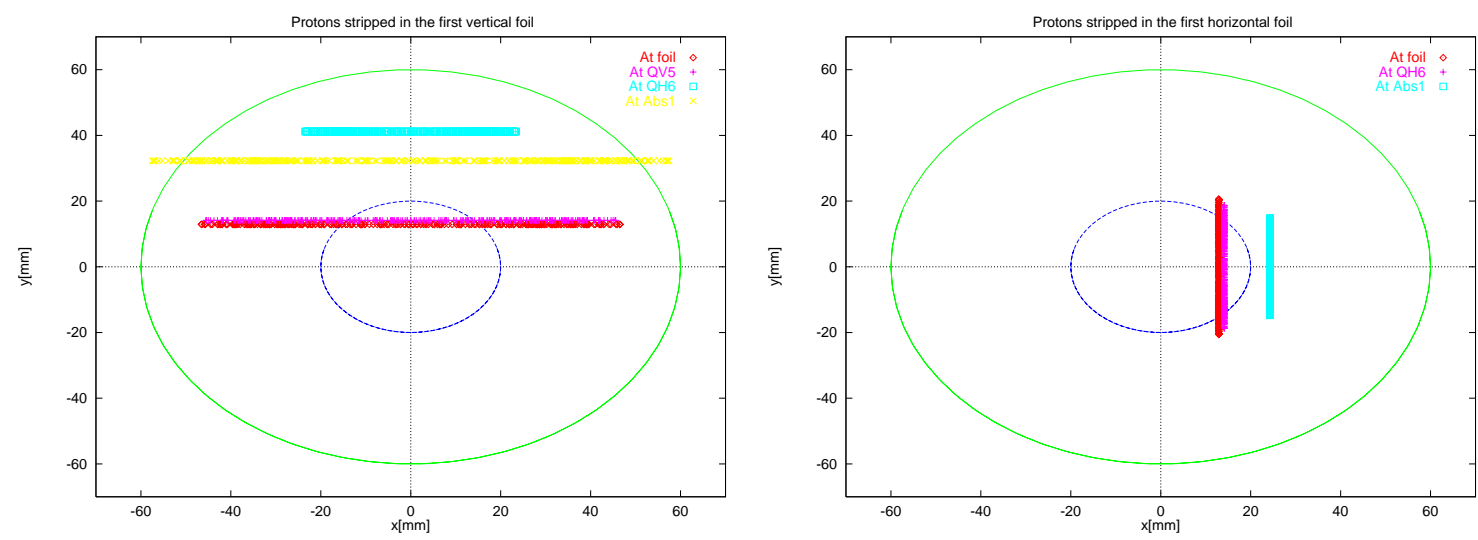

Figure 4: Transverse space projection of the proton beam after scraping in the vertical (left) and horizontal foil (right). The proton beam clears the quadrupoles aperture and hits the absorber with a large impact parameter. 
absorbers was optimized to achieve large impact parameters for both proton beams. The aperture of the collimators has been fixed to $20 \mathrm{~mm}$ radius. The changes required in the layout of the beam line are indicated in Fig. 3. Collimators should be located as close as possible to the end of the drift space to increase the impact parameter and the efficiency.
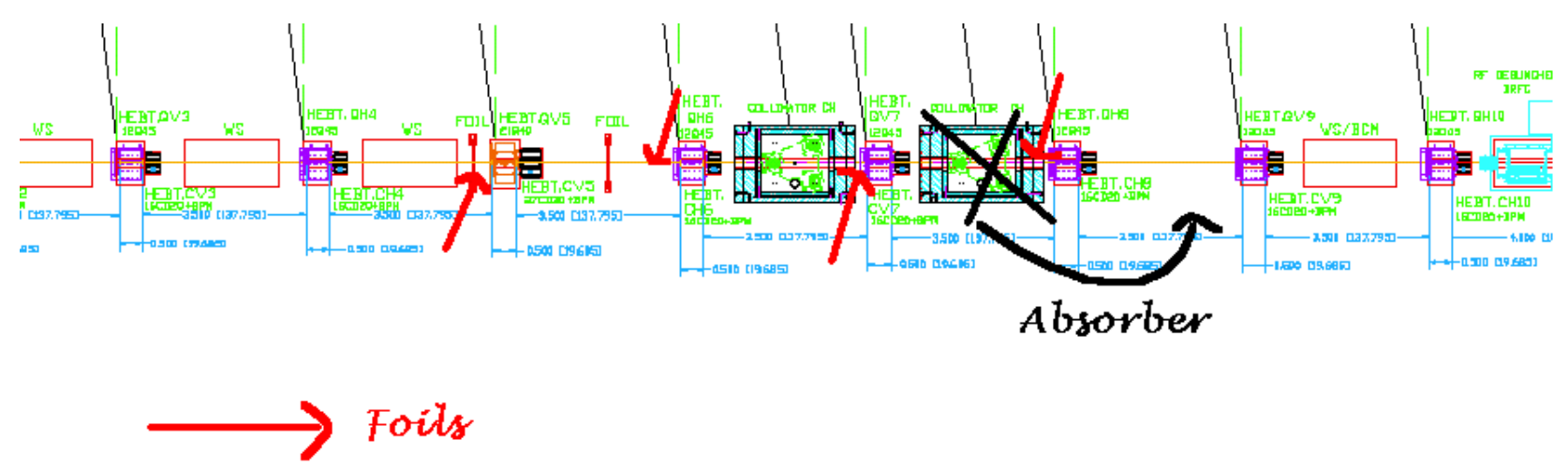

Figure 5: Required changes in the layout of the HEBT collimation area

\subsection{Estimated performance}

For $H^{-}$collimation by charge exchange the interception of the secondary halo by the absorber is $100 \%$ efficient. Still, due to small impact parameters, the protons have a finite probability of escaping the absorber without being removed.

Using the values for the impact parameter on Tab. 1, we simulate the passage of the protons through the material of the absorber with a Monte Carlo code [6]. The absorption efficiency for the secondary $\mathrm{H}^{+}$beam generated at each foil is given in Tab. 1. For efficiency simulations we used a simplified model of the absorbers with a geometry presenting an equivalent mass of stainless steel. A more realistic model is used for secondary particle production and radiation calculations [7].

\begin{tabular}{|c|c|c|c|c|c|}
\hline Name & $\begin{array}{l}\text { Apert. } \\
{[\mathrm{mm}]}\end{array}$ & $\begin{array}{l}\text { Impac } \\
{[\mathrm{mm}]}\end{array}$ & $\begin{array}{l}\text { param. } \\
\text { [mrad] }\end{array}$ & $\begin{array}{l}\text { Eff. } \\
{[\%]}\end{array}$ & Abs \\
\hline Scr 1 U/D & 13 & 12.3 & -5.9 & 94.3 & 1 \\
\hline Scr $1 \mathrm{~L} / \mathrm{R}$ & 17 & 4.3 & 6.7 & 91.1 & 1 \\
\hline Scr 2 U/D & 13 & 15.0 & -4.1 & 95.9 & 2 \\
\hline Scr $2 \mathrm{~L} / \mathrm{R}$ & 17 & 3.3 & 6.1 & 88.7 & 2 \\
\hline
\end{tabular}

Table 1: Aperture, impact parameter and simulated efficiency for every pair foil/absorber. The initial acceptance at the foils correspond to $13 \pi \mathrm{mm} \cdot \mathrm{mrad}$. The efficiency has been calculated for $1 \mathrm{GeV}$ energy protons.

The particles escaping the collimator loose a significant fraction of their total momentum by ionization with the absorber material. They are lost along the achromat located immediately after the last collimator.

These values of efficiency are calculated for the nominal aperture of the foils. Opening or closing them produces a different impact parameter at the absorber and changes the final efficiency. In Fig. 6 
the absorption efficiency is shown as a function of the primary foil acceptance given in $\mathrm{mm} \cdot \mathrm{mrad}$. In the same way, the absorption efficiency was calculated for an energy of $1 \mathrm{GeV}$. Larger energy

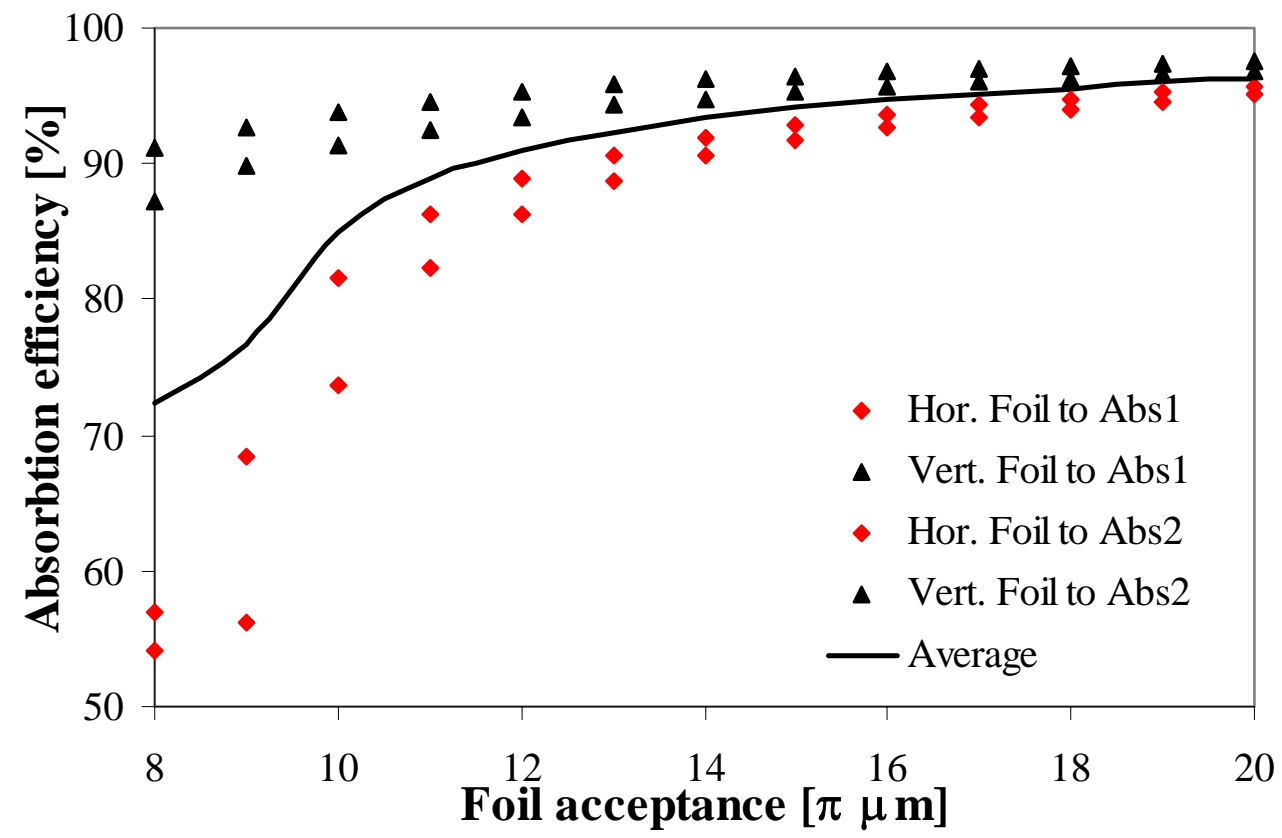

Figure 6: Percentage of protons absorbed in each of the absorbers as a function of foil acceptance. The average from all four foil/absorber sets is plotted in black.

result on slightly better efficiency and a different loss pattern downstream from the collimator. On the other hand, a larger energy beam requires a larger collimator to contain the hadronic shower. A potential upgrade of the collimators should be considered if the final linac energy differs from $1 \mathrm{GeV}$.

\section{HEBT longitudinal collimation}

The tightest requirement for the longitudinal extent of the $H^{-}$beam is given by the RF capture in the ring. Previous studies of the longitudinal dynamics have concluded that any beam above $\pm 4 \mathrm{MeV}$ ends in the gap between consecutive bunches inducing intolerable losses during extraction. A less stringent limit is the momentum acceptance in the HEBT achromat where dispersion is non-zero. These two limits are represented in Fig. 7 by dashed lines.

We thus locate a charge exchange foil in a high dispersion region in the HEBT achromat to remove the longitudinal tails of the beam before entering in the ring. The losses expected in this collimator are much larger than in the transverse case due to the tighter longitudinal acceptance. Also the uncertainty about the energy jitter and momentum spread coming from the superconducting linac translate into a larger beam loss budget.

Because the one-pass condition in a transfer line, only particles with the right betatron phase are intercepted by the foil and striped even if they have large momentum deviation and emittance. The 


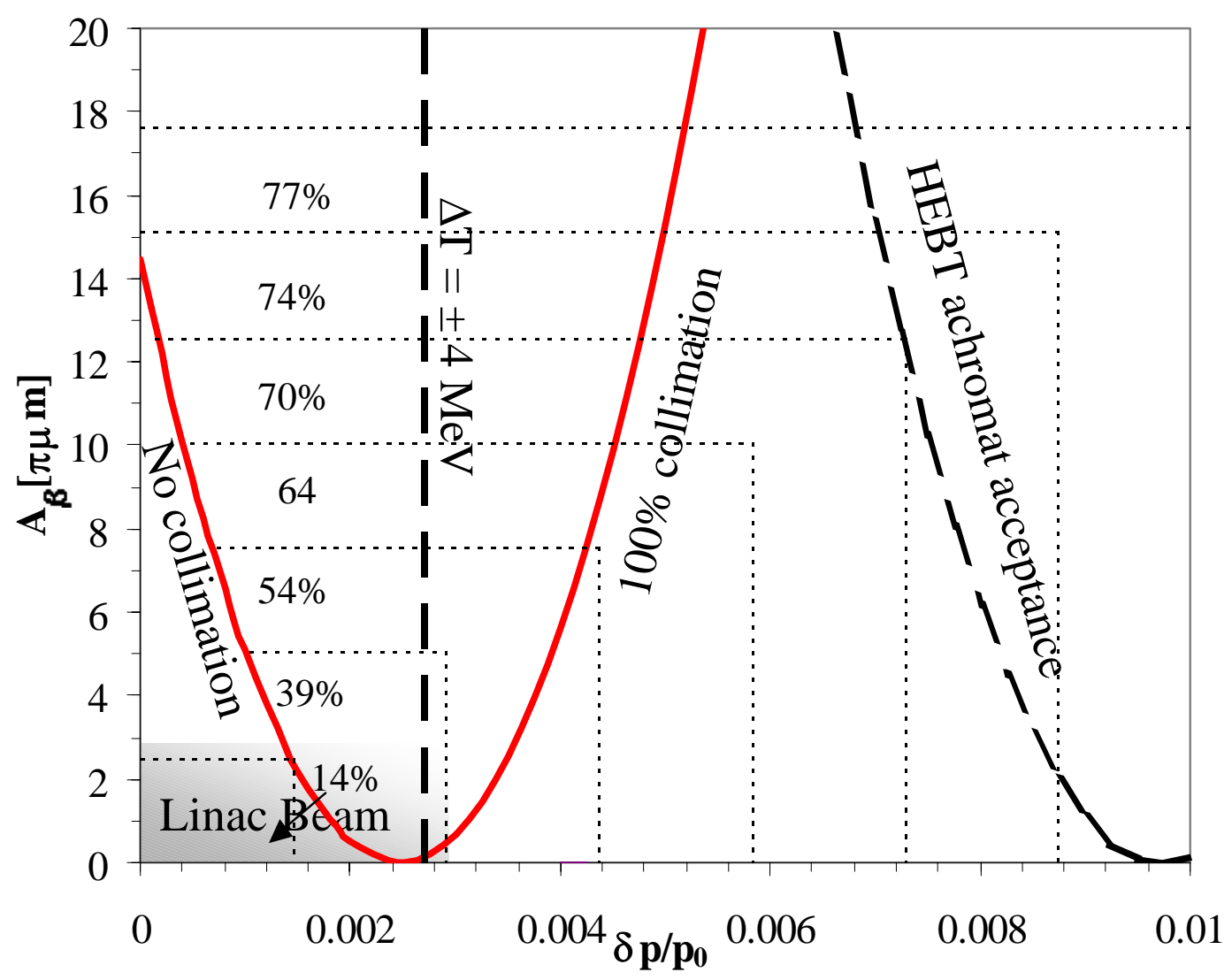

Figure 7: Collimator acceptance in terms of betatron amplitude vs. $\delta p / p_{0}$. The geometry acceptance of the HEBT achromat and the momentum spread limit at $\pm 4 \mathrm{MeV}$ are represented by dashed lines.

adjustable foil shaves the $H^{-}$beam halo when the following condition is fulfilled

$$
x=\sqrt{\epsilon_{x} \cdot \beta} \cdot \sin (\phi)+D \cdot \delta p / p_{0} \geq x_{c u t}
$$

where $x_{c u t}=17 \mathrm{~mm}$ is the foil horizontal position and $\mathrm{D}=6.9 \mathrm{~m}$ and $\beta=20 \mathrm{~m}$ are the dispersion and beta function at the foil.

An acceptance plot is shown in Fig. 7 where the variables are the horizontal emittance and relative momentum deviation of the $H^{-}$ion. The escaping halo clears the HEBT aperture for the emittance range of interest. However, some particles escape with energy deviation above $4 \mathrm{MeV}$ which contribute to the beam in the gap inside the ring. Assuming gaussian distributions, a nominal beam coming from the linac with a transverse emittance of $\epsilon_{r m s}=0.28 \pi \mathrm{mm} \cdot \mathrm{mrad}$ and a energy spread of $\Delta T_{r m s}= \pm 0.72 \mathrm{MeV}$ will have a less than a fraction $10^{-4}$ above the limit determined by the foil. A Montecarlo integration predicts an interception efficiency of the gaussian tails only of $14 \%$. For a beam with an emittance and momentum deviation twice the nominal values the efficiency increase to $39 \%$. However, large momentum tails are removed more efficiently than large emittance particles. The interception efficiency for particles with momentum deviation larger than $4 \mathrm{MeV}$ is better than $90 \%$ ensuring that the population of the gap in the ring is less than $\approx 10^{-5}$ of the total beam from the nominal linac values.

After being striped, the proton beam is deviated by the dipole field into a large absorber outside 
the circulating beam trajectory. The vacuum pipe geometry after the foil has been adjusted to clear the dipoles and drive the $\mathrm{H}^{+}$beam on the absorber [8]. In this case, the absorption efficiency in the collimator is $100 \%$, as protons are deviated from the main path and hit the absorber located outside the accelerator.

\section{REFERENCES}

[1] D. Raparia et al., " The NSNS High Energy Beam Transport Line” PAC 1997, Vancouver

[2] H. Ludewig et al., "Design of the NSNS Collimator System". BNL/NSNS Technical Note 18. March 1997

[3] H. Ludewig et al., "Revised Conceptual Design for the Collimator". BNL/NSNS Technical Note 44. April 1998

[4] H. Ludewig et al., "Collimator Systems for the SNS Ring". PAC 1999, New York, 1999

[5] D. Raparia, J. Alessi and Y.Y. Lee, "Collimation in the HEBT". BNL/NSNS Technical Note 65. August 1999

[6] J.B. Jeanneret and T. Trenkler "K2: A Software Package Evaluating Collimation Systems in Circular Colliders". CERN SL 94-105 AP.

[7] H. Ludewig, "Preliminary Estimate of Dose Following Machine Shutdown From Collimators, Vacuum Chamber Walls, and Adjacent Magnets" BNL/SNS Technical Note 67. October 1999

[8] P. He et al., "HEBT Momentum Scraper, H+ Ray Trace Simulation and Vacuum Chamber Design" PAC 2001 Chicago 2001 